Journal of Mathematics and Statistics 2 (2): 373-377, 2006

ISSN 1549-3644

(C) 2005 Science Publications

\title{
A Branching Cell Population Model Allowing for Cell Death
}

\author{
Mounir Aout \\ Département STID Lisieux, IUT de Caen \\ 11,bd Jules Ferry, 14100 Lisieux, France
}

\begin{abstract}
We use multi-type branching processes to describe a general cell population model allowing for cell death. Unlike the case without cell death, the process can be subcritical, critical or supercritical. Since we are interested in the supercritical case where the process can escape extinction with positive probability, we give conditions ensuring the supercriticality. In this context, we show the existence of the Malthusian parameter and the stable birth-type distribution which we get analytically under additional assumptions.
\end{abstract}

Key words: Branching Processes. Malthusian parameter. Stable type Distribution. Cell model.

\section{INTRODUCTION}

For understanding the dynamics of cell populations, and responding to the problems concerning this field of study, there is a need for mathematical models. Many such models have been proposed in the literature. Most of these are deterministic and formulated in terms of partial differential equations.

A recent, extensive list of references, and a nice collection of articles on different kinds of cell population models can be found in Arino ${ }^{[3]}$ and Arino et al. ${ }^{[4]}$ and reference therein. In fact, we can not pass over without pointing out the paper of Bell and Anderson $^{[5]}$ which gave rise to the so-called BellAnderson model. For a recent account of the stochastic approach, we refer to ${ }^{[9]}$ and ${ }^{[1]}$. Taib ${ }^{[9]}$ described a stochastic version of Bell-Anderson model and discussed the existence of the stable type distribution. $\mathrm{He}$ assumed equal cell division and disregarded cell death. In Alexandersson ${ }^{[1]}$, a quite general model was presented assuming unequal cell division. Moreover, the stable type distribution was given explicitly by adding an extra assumption of a critical size that each cell has to pass before division, called the nonoverlapping case. One of the features of these works is that cells are not allowed to die. Since cell loss due to cell death is known to play an important role in cell kinetics, we propose to augment Alexandersson's model by adding cell death.

The main object of interest is to prove the existence of the Malthusian parameter such that the process is supercritical. Furthermore, we show the existence of the stable type distribution which plays an important role in the asymptotic composition of our model.

\section{MATERIALS AND METHODS}

Let $(S, \mathfrak{I})$ be a measurable space with $\mathfrak{I}$ countably generated and $v(r, d s \times d t)$ a non-negative kernel on

$\left(S \times R_{+}, \mathfrak{I} \times \mathfrak{R}\right)$, where $\mathfrak{R}$ is the Borel $\sigma$-algebra on $R_{+}$.

The basis $v^{*}$ of $v$ is defined by:

$$
v^{*}(x, d y)=\int_{R_{+}} v(x, d y \times d t)
$$

\section{Definition1.}

The Perron root of $v^{*}$, denoted by $\rho\left(v^{*}\right)$, is defined by

$$
\rho\left(v^{*}\right)^{-1}=\sup \left\{\lambda \in R_{+} ; \sum_{n \geq 0} \lambda^{n} v^{* n} \text { is } \sigma-\text { finite }\right\}
$$

where $v^{* n}(x, A)=\int_{R_{+}} v^{* n-1}(x, d s) v^{*}(s, A)$

Next define for $\alpha \geq 0$

$$
v_{\alpha}^{*}(x, d y)=\int_{R_{+}} e^{-\alpha t} v(x, d y \times d t)
$$

It is asked under which conditions there exists a number $\alpha$ such that $\rho\left(v_{\alpha}^{*}\right)=1$.

Such a number $\alpha$ is called the Malthusian parameter. The process is subcritical, critical or supercritical according to $\alpha<0, \alpha=0$ or $\alpha>0$.

\section{Definition2.}

The kernel $\rho\left(v_{\alpha}^{*}\right)$ is called conservative if there exists a $\sigma$-finite non-zero measure $m$ on $(S, \mathfrak{I})$ such that

Corresponding Author: Mounir Aout, Département STID, IUTde Caen, 11 Bd Jules Ferry, 14100 Lisieux, France 


$$
m(A)>0 \Rightarrow \sum_{n \geq 0} v_{\alpha}^{* n}(x, A)=\infty, \quad A \in \mathfrak{I}
$$

Once the population is Malthusian and supercritical, this meaning that there is a number $\alpha>0$ such that

$\rho\left(v_{\alpha}^{*}\right)=1$ and $v_{\alpha}^{*}$ is conservative, we will be able to give an abstract version of the Perron-Frobenius Theorem $^{[8]}$.

\section{Theorem1.}

When $v_{\alpha}^{*}$ is conservative in the above sense, it follows that there exists a $\sigma$-finite measure $\pi$ on $(S, \mathfrak{I})$ and a positive, finite $(\pi$-a.e.), $\mathfrak{I}$-measurable function $h$ such that

$$
\begin{gathered}
\int_{S} v_{\alpha}^{*}(x, A) \pi(d x)=\pi(A) ; \quad A \in \mathfrak{I} \\
\int_{S} v_{\mathscr{\alpha}}^{*}(x, d y) h(y)=h(x) ; \quad x \in S
\end{gathered}
$$

and $\nu_{\alpha}^{*}$ is $\pi$-conservative.

The measure and the function are unique up to multiplicative constants.

In the sequel these two objects are assumed to be normalized according to

$$
\int_{S} \pi(d s)=1 \text { and } \int_{S} h(s) \pi(d s)=1
$$

A simple but crucial criterion for conservativity is presented in ${ }^{[9]}$.

\section{Lemma1.}

A sufficient condition for $v_{\alpha}^{*}$ to be conservative is the existence of a $\sigma$-finite non-zero measure $m_{\alpha}$ on $(S, \mathfrak{I})$ such that $v_{\tilde{\alpha}}^{*}(r, A) \geq m_{\alpha}(A), \forall r \in S, A \in \mathfrak{I}$

\section{MATHEMATICAL MODEL}

\section{The reproduction kernel}

In this section we describe a quite general cell population model based on ${ }^{[1]}$. Our population consists of cells, which inherit types at birth, grow according to a cell-size determined intensity and split into two daughter cells or die without leaving any progeny. Here the type is the birth size of the cell (e.g. mass, volume, DNA-content etc). The Ulam-Harris set of all conceivable cells is denoted by

$$
I=\{0\}\{1,2\}^{n}
$$

where $\{0\}$ is the zeroth generation consisting of the founder cell. The type space is an interval $S=(0, M)$ on the real line. A cell with birth size $r \in S$ chooses a life career $\omega \operatorname{from}(\Omega, A)$ using $P(r,$.$) , the life law of$ cells of type $r$. We construct the population space $\left(S \times \Omega^{I}, \mathfrak{I} \times A^{I}\right)$, and as explained in ${ }^{[6]}$, there exists a unique probability measure $P_{r}$ on the entire population process, where $r \in S$ is the type of the ancestor.

At birth a cell has an initial size $r$, grows to reach the size $m(r, t)$ at age $t$. The cell growth is taken to be deterministic according to the growth equation $\frac{d m}{d t}=g(m)$ where $g$ is continuous and strictly positive on $(0,2 M)$. When division occurs at a certain size dependant rate $b(s), s \in S$, the cell divides into the fractions $\gamma$ and $1-\gamma$, where $\gamma$ is a random variable in $(0,1)$ with density function $f_{\gamma}(m(r, \lambda), p)$,

$p_{1} \leq p \leq p_{2}$ where $p_{2}=1-p_{1} \in(0,1)$ depends on the size at cell division. We will assume that $\gamma$ is symmetrically distributed around $1 / 2$ and that $f_{\gamma}$ is unimodal, i.e. $E_{r}[\gamma]=1 / 2$, for all $r \in S$ and $f_{\gamma}(m, p)=f_{\gamma}(m, 1-p)$.

The basic assumption is that, besides the non-negative splitting intensity $b(s)$, there is a non-negative death intensity $d(s)$. The time to division or to death are denoted by $\lambda_{1}$ and $\lambda_{2}$ respectively, and

$$
\begin{aligned}
& P_{r}\left(\lambda_{1}>t\right)=e^{-\int_{0}^{t} b(m(r, u)) d u} \\
& P_{r}\left(\lambda_{2}>t\right)=e^{-\int_{0}^{t} d(m(r, u)) d u}
\end{aligned}
$$

It follows that the probability that a cell with type $r$ splits during an infinitesimally small interval is

$$
P_{r}\left(\lambda_{1} \in d t, \lambda_{1}<\lambda_{2}\right)=b(m(r, t)) e^{\int^{t}(b(m(r, u))+d(m(r, u))) d u}
$$

We will also need the following notations where $r, x \in S$ :

$$
T(x)=\int_{0}^{x} \frac{1}{g(y)} d y, \quad C(x)=\int_{0}^{x} \frac{b(y)+d(y)}{g(y)} d y
$$

and

$$
q(x, s)=\frac{b(x)}{x g(x)} f_{\gamma}\left(x, \frac{s}{x}\right)
$$

$T(x)-T(r)$ is the time it takes for a cell to grow from size $r$ at birth to size $x$.

To see this we make a change of variable $y=m(r, t)$ in $T(x)-T(r)=\int_{r}^{x} \frac{1}{g(y)} d y$ we will get 
$\int_{r}^{x} \frac{1}{g(y)} d y=\int_{0}^{u} \frac{g(m(r, t))}{g(m(r, t))} d t=u$ where $u$ is the time it takes for a cell to grow from size $r$ at birth to size $x$, thus $T(x)-T(r)$ is precisely this time.

The basic parameter of the process is the so-called the reproduction kernel $\mu(r, d s \times d t)$ which gives the expected number of children with birth sizes in $d s$ born to a cell of type $r$ in the age interval $d t$ :

$$
\begin{aligned}
\mu(r, d s \times d t) & =E_{r}\left[1_{\left\{\lambda_{1}<\lambda_{2}, \lambda_{1} \in d t\right\}} 1_{\left\{\gamma m\left(r, \lambda_{1}\right) \in d s\right\}}\right. \\
& \left.+1_{\left\{\lambda_{1}<\lambda_{2}, \lambda_{1} \in d t\right\}} 1_{\left\{(1-\gamma) m\left(r, \lambda_{1}\right) \in d s\right\}}\right] \\
& =2 \int_{r}^{2 M} 1_{\{\mathrm{T}(\mathrm{x})-\mathrm{T}(\mathrm{r}) \in \mathrm{dt}\}} q(x, s) e^{-(C(x)-C(r))} d x d s
\end{aligned}
$$

so that

$$
\begin{gathered}
\mu^{*}(r, d s)=2 \int_{r}^{2 M} q(x, s) e^{-(C(x)-C(r))} d x d s \\
\mu^{*}(r, S)=2 e^{C(r)} \int_{r}^{2 M} \frac{b(x)}{g(x)} e^{-C(x)} d x \\
\mu_{\alpha}^{*}(r, d s)=2 \int_{r}^{2 M} q(x, s) e^{-\alpha(T(x)-T(r))-(C(x)-C(r))} d x d s
\end{gathered}
$$

In the sequel we will be assumed that a cell has to divide or die before reaching size $2 M$.

\section{The stable type distribution.}

In this section we will prove the existence of the stable type distribution. We will first start by giving an example to give an idea how this framework can be used:

\section{Example1.}

Assume that $\forall s, b(s)=b, d(s)=d$ and $g(s)=g$. We can show easily that:

$\mu^{*}(r, S)=2$

$\mu_{\alpha}^{*}(r, d s)=\frac{2 b}{b+d+\alpha}$

The solution of the equation $\rho\left(\mu_{\alpha}^{*}\right)=1$ is $\alpha=b-d$.

To ensure the supercriticality, we need the condition $b>d$.

Let us turn to the general case. We give our main result.

\section{Theorem2.}

Under the assumptions stated on our reproduction kernel $\mu$, the Malthusian parameter $\alpha$ exists and $\mu_{\alpha}^{*}$ is conservative.

\section{Proof.}

By the continuity of the Laplace transform and that of
Perron roots as demonstrated in ${ }^{[1]}$ and which we apply here, we have $\rho\left(\mu_{\alpha}^{*}\right) \rightarrow+\infty$ as $\alpha \rightarrow-\infty$ and $\rho\left(\mu_{\alpha}^{*}\right) \rightarrow 0$ as $\alpha \rightarrow+\infty$. The mean value theorem implies that there exists an $\alpha$ such that $\rho\left(\mu_{\alpha}^{*}\right)=1$. Hence the Malthusian parameter exists and it can take any value in $R$.

Unlike the case without cell death, our model can be subcritical, critical or supercritical depending on the sign of $\alpha$, and then on how $b$ and $d$ interact. Since we are interested in the supercritical case, we need to find a condition ensuring the supercriticality. For this, notice that in the case where $b(s)=d(s)$ we have:

$$
\mu^{*}(r, S)=2 e^{C(r)} \int_{r}^{2 M} \frac{b(x)}{g(x)} e^{-C^{*}(x)} d x
$$

$$
=1
$$

where $C^{*}(x)=\int_{0}^{x} \frac{2 b(y)}{g(y)} d y$,

then $\rho\left(\mu^{*}\right)=1$ and $\mu^{*}$ must be critical. We thus see that the process is supercritical if for example $\inf _{s \in S} \frac{b(s)}{d(s)}=\eta>1$ in which case $\rho\left(\mu^{*}\right) \geq \frac{2 \eta}{\eta+1}$.

Conservativity is all that is needed to guarantee the existence of a stable size distribution. In our case, using lemma1, it is enough to exhibit a candidate which can play the role of the measure cited above. It turns out that it is possible to take:

$$
m_{\alpha}(A)=2 \int_{M}^{2 M} \int_{A} q(x, s) e^{-\alpha T(x)-C(x)} d x d s
$$

Since

$$
\begin{aligned}
\mu_{\alpha}^{*}(r, A) & =2 \int_{r}^{2 M} \int_{A} q(x, s) e^{-\alpha(T(x)-T(r))-(C(x)-C(r))} d x d s \\
& \geq 2 \int_{M}^{2 M} \int_{A} q(x, s) e^{-\alpha T(x)-C(x)} d x d s \\
& =m_{\alpha}(A)
\end{aligned}
$$

\section{The nonoverlapping case}

This section is concerned with the explicit expression of the stable type distribution. Once we have established the existence of the Malthusian parameter $\alpha$ such that $\mu_{\alpha}^{*}$ is conservative, we know from Theorem 1 that there exist a function $h$ and a measure $\pi$. The problem is then to solve the following equations

$$
h(r)=\int_{S} \mu_{\alpha}^{*}(r, d s) h(s) ; \quad r \in S
$$




$$
\pi(A)=\int_{S} \mu_{\alpha}^{*}(r, A) \pi(d r) ; \quad \mathrm{A} \in \mathfrak{I}
$$

where

$$
\mu_{\alpha}^{*}(r, d s)=2 \int_{r}^{2 M} q(x, s) e^{-\alpha(T(x)-T(r))-(C(x)-C(r))} d x d s
$$

This can be done by using some suitable numerical methods. In some special cases, for example the case treated in Examplel, the solutions can be obtained explicitly. However, in order to keep our model more general and without having to assume specific distributions and linear growth, we will add the assumption of a critical size to our cell model. We assume that there is a critical size $m_{0}$ such that a new born cell always has size less than $m_{0}$, and then it grows passed $m_{0}$ before it divides. The type space becomes $S=\left(0, m_{0}\right)$ and the hazard rate function $b$ is defined on $\left(m_{0}, 2 m_{0}\right)$.

The kernel becomes

$$
\mu_{\alpha}^{*}(r, d s)=2 \int_{m_{0}}^{2 m_{0}} q(x, s) e^{-\alpha(T(x)-T(r))-(C(x)-C(r))} d x d s
$$

By iterating we get

$$
\begin{aligned}
\mu_{\alpha}^{* \mathrm{n}}(r, d s) & =\mu_{\alpha}^{*}(r, d s)\left(\int_{S} \mu_{\alpha}^{*}(x, d x)\right)^{n-1} \\
& =\mu_{\alpha}^{*}(r, d s) I^{n-1}(\alpha)
\end{aligned}
$$

Note that this iteration does not hold without the above assumption.

We search $\alpha$ such that the Perron root is 1 for $\mu_{\alpha}^{*}$. Since $I$ is a non-negative, non-increasing continuous function of $\alpha$ and $I(\alpha) \rightarrow 0$ as $\alpha \rightarrow+\infty$, we can choose $\alpha$ such that $I(\alpha)=1$.

For $\lambda \geq 0$ we have

$$
\begin{aligned}
\sum_{n \geq 0} \lambda^{n} \mu_{\alpha}^{* n}(r, d s) & =\mu_{\alpha}^{*}(r, d s) \sum_{n \geq 0} \lambda^{n} I^{n-1}(\alpha) \\
& =\mu_{\alpha}^{*}(r, d s) \sum_{n \geq 0} \lambda^{n}
\end{aligned}
$$

This sum converges for $\lambda<1$ and diverges for $\lambda \geq 1$ and then the Perron root is 1 and $\alpha$ is the Malthusian parameter.

Let us turn to prove the conservativity of the kernel $\mu_{\alpha}^{*}$.

\section{Theorem3.}

The kernel $\mu_{\alpha}^{*}$ is conservative.

Proof. We have

$$
\mu_{\alpha}^{*}(r, A)=2 \int_{m_{0}}^{2 m_{0}} \int_{A} q(x, s) e^{-\alpha(T(x)-T(r))-(C(x)-C(r))} d x d s
$$

The non-zero measure $m_{\alpha}$, defined as

$$
m_{\alpha}(A)=2 \int_{m_{0} A}^{2 m_{0}} \int_{A} q(x, s) e^{-\alpha T(x)-C(x)} d x d s
$$

is a minorizing measure of the kernel $\mu_{\alpha}^{*}$.

Moreover we have for all $r \in S$

$$
\begin{aligned}
\sum_{n \geq 0} \mu_{\alpha}^{* n}(r, A) & =\sum_{n \geq 0} \mu_{\alpha}^{*}(r, A) I^{n-1}(\alpha) \\
& =\sum_{n \geq 0} \mu_{\alpha}^{*}(r, A)
\end{aligned}
$$

and this sum is infinite whenever $\mu_{\alpha}^{*}(r, A)>0$.

Thus this kernel is conservative since for all $r \in S$

$m_{\alpha}(A)>0 \Rightarrow \mu_{\tilde{\alpha}}^{*}(r, A)>0$.

We are now able to apply Theorem1. It follows that there exist a $\sigma$-finite measure $\pi$ and a non-negative $\pi$-a.e. finite function $h$ such that

$$
\begin{gathered}
h(r)=\int_{S} \mu_{\alpha}^{*}(r, d s) h(s) ; \quad r \in S \\
\pi(A)=\int_{S} \mu_{\alpha}^{*}(r, A) \pi(d r) ; \quad \mathrm{A} \in \mathfrak{I}
\end{gathered}
$$

From the same theorem we also know that these equations determine $h$ and $\pi$ uniquely up to multiplicative constants, but we want $h$ and $\pi$ to satisfy the conditions

$$
\int_{S} \pi(d s)=1 \text { and } \int_{S} h(s) \pi(d s)=1
$$

Solving these equations we get

$$
\begin{aligned}
h(r) & =e^{\alpha T(r)+C(r)} \int_{S} h(s) 2 \int_{m_{0}}^{2 m_{0}} q(x, s) e^{-\alpha T(x)-C(x)} d x d s \\
& =c_{1} e^{\alpha T(r)+C(r)}
\end{aligned}
$$

Moreover

$$
\begin{aligned}
\pi(A) & =\int_{S} \int_{A} 2 \int_{m_{0}}^{2 m_{0}} q(x, s) e^{-\alpha(T(x)-T(r))-(C(x)-C(r))} d x d s \pi(d r) \\
& =\int_{A} 2 \int_{m_{0}}^{2 m_{0}} q(x, s) e^{-\alpha T(x)-C(x)} d x d s \int_{S} e^{\alpha T(r)+C(r)} \pi(d r) \\
& =c_{2} \int_{A} 2 \int_{m_{0}}^{2 m_{0}} q(x, s) e^{-\alpha T(x)-C(x)} d x d s
\end{aligned}
$$

To determine $c_{1}$ and $c_{2}$ we use conditions stated in equation 1 and it is nor difficult to verify that 


$$
\begin{aligned}
\int_{S} h(s) \pi(d s) & =2 \int_{S} c_{1} e^{\alpha T(s)+C(s)} c_{2} \int_{m_{0}}^{2 m_{0}} q(x, s) e^{-\alpha T(x)-C(x)} d x d s \\
& =c_{1} c_{2} \int_{S} 2 \int_{m_{0}}^{2 m_{0}} q(x, s) e^{-\alpha(T(x)-T(s))-(C(x)-C(s))} d x d s \\
& =c_{1} c_{2} \int_{S} \mu_{\alpha}^{*}(s, d s) \\
& =c_{1} c_{2} I(\alpha) \\
& =c_{1} c_{2}
\end{aligned}
$$

Thus

$$
\begin{aligned}
c_{1} & =\left(c_{2}\right)^{-1} \\
& =\int_{S} 2 \int_{m_{0}}^{2 m_{0}} q(x, s) e^{-\alpha T(x)-C(x)} d x d s
\end{aligned}
$$

\section{ASYMPTOTIC COMPOSITION}

Once the existence of the Malthusian parameter is proved and the expression of the stable type distribution is given, we will be able to derive various other asymptotic distributions concerning cell populations using the framework of branching processes counted by random characteristics ${ }^{[7]}$ with respect to some property. Examples of such distributions are the $\alpha$-curve, the proportion of cells in a particular phases etc. For the illustration, we give the expression of the $\alpha$-curve .

\section{The $\alpha$-curve}

The $\alpha$-curve is the graph of the function $\alpha(t)$ describing the proportion of cells still undivided at age $t$ as a function of $t$. An alternative interpretation ${ }^{[7]}$ is that $\alpha(t)$ is the probability that the age at death or division of a sampled cell at random, called ego, is larger than $t$. Using an appropriate random characteristic with simple manipulations, that is

We know from ${ }^{[7]}$ that

$$
\chi(u)=1_{\left\{R_{+}\right\}}(u) 1_{\left\{\lambda_{1}>t, \lambda_{2}>t\right\}}
$$

$$
\begin{aligned}
\alpha(t) & =E_{\pi}\left[\chi^{*}(\alpha)\right] \\
& =\int_{S} E_{r}\left[\int_{0}^{\infty} \alpha e^{-\alpha t} \chi(u) \pi(d r)\right] \\
& =\int_{S} P_{r}\left(\lambda_{1}>t, \lambda_{2}>t\right) \pi(d r) \\
& =\int_{S} e^{-\int^{t}(b(m(r, v))+d(m(r, v))) d v} \pi(d r)
\end{aligned}
$$

\section{DISCUSSION}

In this paper, we proposed a quite general model dealing with cell death. We showed the existence of the Malthusian parameter and gave an explicit expression of the stable type distribution. Once these objects were settled, one could study various asymptotic properties of the model. In addition, it will be a considerable gain if we find some experimental data to validate our model and calibrate the parameters. This is one of the options of future research. Another option is to deal with the stochastic growth. On the other hand, our goal was to propose a quite general model by adding cell death to cover a large class of cell populations. Nevertheless, some models which take quiescence into account are not covered by our model. This problem was treated in ${ }^{[2]}$ in some special cases.

\section{REFERENCES}

1. Alexandersson, M., 1999. Branching Processes and Cell Populations. Doctoral Thesis. Chalmers U. Tech. and Gothenburg U.

2. Aout, M., 2000. Branching Processes Cell Population Models. Doctoral thesis. Dep. Mathematics. Sidi Mohamed BenAbdellah U.

3. Arino, O., 1995. A Survey of Structured Cell Population Dynamics. Acta Biotheoretica, 43: 3-25

4. Arino, O., D. Axelord, and M. Kimmel, editors., 1997. Advances in Mathematical Population Dynamics- Molecules, Cells and Man. World Scientific.

5. Bell, G.I. and E.C. Anderson, 1967. Cell Growth and Division I: A mathematical model with applications to cell volume distributions in mammalian suspension cultures. Bioph. J., 7: 329-351

6. Jagers, P., 1989. General Branching Processes as Markov Fields. Stoch. Proc. Appl., 32: 183-242.

7. Jagers, P. and O. Nerman, 1996. The Asymptotic Composition of Supercritical Multi-type Branching Populations. Mar Yor, ed., Seminaire de Probabilités. Lectures Notes In Mathematics. Springer Verlag

8. Shurenkov, V.M., 1989. Ergodicheskie protsessy markova. Nauka.

9. Taib, Z., 1999. A Branching Process Version of the Bell- Anderson Cell Population Model. Stoch. Models. 4: 719-730. 